\title{
Stochastic Methods in Atomic Systems and QED
}

\author{
R. F. O'Connell
}

\begin{abstract}
We show that treating the blackbody radiation field as a heat bath enables one to utilize powerful techniques from the realm of stochastic physics (such as the fluctuation-dissipation theorem and the related radiation damping) in order to treat problems that could not be treated rigorously by conventional methods. We illustrate our remarks by discussing specifically the effect of temperature on atomic spectral lines, and the solution to the problem of runaway solutions in the equation of motion of a radiating electron. We also present brief discussions relating to anomalous diffusion and wave packet spreading in a radiation field and the influence of quantum effects on the laws of thermodynamics.

PACS number(s): 31.30.jg, 05.40.-a
\end{abstract}

\section{Introduction}

Stochastic physics deals with fluctuations, principally thermal and quantum. The subject is often loosely referred to as "Brownian motion" since it was first seriously studied when Brown, in 1828, observed the random motion of pollen grains immersed in a fluid [1] at temperature $T$, in the absence of external forces. Einstein [2] used a diffusion equation and showed that, for large times, the mean-square displacement is proportional to $T / \gamma$, where $T$ is the temperature and $\gamma^{-1}$ is the collision rate, this being the first example of a fluctuation - dissipation (FD) theorem. Soon after, Langevin [3] presented a simple phenomenological approach, by writing down the first example of a stochastic differential equation. In this equation, the total force acting on a particle due to its environment is separated into two parts: a frictional force and a fluctuation (random) force. These terms are very different in nature: The fluctuation term is basically microscopic in nature and has a time scale determined by the mean time between collisions whereas the time scale of the frictional force is proportional to the self-diffusion constant and is much larger.

Later, another example of a fluctuation-dissipation theorem, arose in the analysis of so-called Johnson-Nyquist noise [4, 5]. All of this work was classical in nature but in 1951, Callen and Welton [6] presented a general quantum FD theorem. Such a theorem is implicit in the pioneering work of Ford, Kac and Mazur [7] who presented a microscopic quantum Langevin approach to the case of an oscillator interacting with a heat bath composed of an infinite number of coupled harmonic oscillators. This work was later generalized by Ford and Kac [8] and by Ford et al. [9]. In the latter paper, the earlier work was generalized by writing down what we referred to as the IO (independent oscillator) model, describing the system of a quantum oscillator in an arbitrary potential, at an arbitrary temperature $T$, interacting with a heat bath of oscillators which were not interacting with each other. By assigning arbitrary masses and frequencies to all the oscillators, we obtained in essence a model which incorporated a variety of existing models. In particular, by means of a series of unitary transformations, we showed

R. F. O'Connell. Department of Physics and Astronomy, Louisiana State University, Baton Rouge, Louisiana 70803-4001

(e-mail: oconnell@phys.lsu.edu) that the blackbody radiation field (BBR) could be treated as a special case of the IO model. Concomitantly, because the Hamiltonian for an oscillator in a BBR field is universally accepted, it provided in essence a "rosetta stone" in validating our choice of the more general IO model rather than any of the various models that one finds in the quantum optics literature. In particular, we showed [9] that the well-known RWA (rotating - wave approximation) model of a heat bath has a serious problem in that the corresponding Hamiltonian does not have a lower bound.

In this paper, we confine our attention, for the most part, to the BBR bath. Thus, in Sec. 2, we discuss the fundamentals underlying this subject and how they may be applied to the experimental results on the effect of temperature on spectral lines. In Sec. 3, we show how the quantum Langevin equation developed in Sec. 2 forms the basis of a derivation of a solution to the problem of runaway solutions. In Sec. 4 , we consider some miscellaneous applications. In particular, we show how motion in a BBR field gives rise to anomalous diffusion and wave packet spreading, phenomena that are not apparently amenable to conventional QED techniques. Also, we point out that thermodynamic concepts developed in Sec. 2 form the basis of arguments against the various claims that quantum affects could lead to violations of the fundamental laws of thermodynamics. In Sec. 5, we present our conclusions.

\section{QED shifts due to blackbody radiation}

Hollberg and Hall [10], using high-precision laser spectroscopy, measured photon heated $\mathrm{Rb}$ atoms to temperatures $T$ as high $1000 K$ and analyzed the photon spectra associated with transitions from the high Rydberg 36s state to the tightly bound $5 \mathrm{~s}$ state. They found an increase in photon energies proportional to $T^{2}$ which they concluded represented energy shifts due to temperature. Our conclusion $[11,12,13]$ is that they have measured free energy shifts, as we will now argue.

There is general agreement that the main frequency shift arises from $T$ effects on the high Rydberg state since the effect of $T$ on the tightly bound state is negligible. In essence, we are dealing with a temperature dependent Lamb shift. Conventional atomic approaches to the problem have been carried out $[14,15]$ leading to the conclusion that the dominant energy shift $\sim T^{2}$. The essence of the conventional calculation can be 
simply obtained by an extension of Welton's $T=0$ calculation [16] for the Lamb shift, and it also serves as a transparent foil to our approach. In this approach the weakly bound Rydberg electron is treated as a free electron which undergoes rapid oscillations due to the electric field associated with the BBR. The energy of oscillation $W(\omega)$ of an electron moving in one dimension in an electric field $E_{0} e^{-i \omega t}$ is

$W(\omega)=e^{2} E_{0}^{2} / 4 m \omega^{2}=\left(2 \pi e^{2} / 3 m \omega^{2}\right)\left(3 E_{0}^{2} / 8 \pi\right)$.

Identifying $3 E_{0}^{2} / 8 \pi$ with $u(\omega, T)$, the energy energy density of the electromagnetic field, one substitutes the Planck distribution

$$
u(\omega, T)=\left(\hbar \omega^{3} / \pi^{2} c^{3}\right) /[\exp (\hbar \omega / k T)-1]
$$

and integrates over all frequencies to obtain the mean energy. In three dimensions this is to be multiplied by a factor of three to give

$U(T)=3 \int_{0}^{\infty} d \omega W(\omega)=\frac{\pi e^{2}(k T)^{2}}{3 \hbar m c^{3}}=\frac{\pi \alpha}{3 m c^{2}}(k T)^{2}$.

This theoretical result appears to agree with experiment. However, as we have previously indicated [12], there are flaws with this analysis since:

(a) missing from (3) is the equipartition term $k T / 2$, which is the leading and dominant term,

(b) radiation damping. The key point is that an atom interacting with BBR at temperature $T$ is a thermodynamic system. Equilibrium is preserved by virtue of the fact that the BBR not only gives energy to the atom but also receives energy from the atom because of dissipative effects [17, 18], which is a beautiful example of the fluctuation - dissipation at work (and analogous to Langevin's treatment of Brownian motion, when he used the reverse process to introduce a fluctuation force to counteract the dissipative force exerted by the fluid). The conclusion is that thermodynamic principles must be used in this atomic problem. In particular, the work done in an isothermal transition (in this case, the energy supplied by a photon driving a transition from the ground state to an excited state) is equal to the change in free energy. Thus, it is our basic contention that the Hollberg - Hall experiment is actually measuring changes in free energy, as distinct from changes in energy. Thus, we next turn to how such changes are calculated. Our starting point is the Hamiltonian of the IO system [11]

$$
\begin{aligned}
H= & \frac{p^{2}}{2 m}+V(x)+\sum_{j} \\
& \left(\frac{p_{j}^{2}}{2 m_{j}}+\frac{1}{2} m_{j} \omega_{j}^{2}\left(q_{j}-x\right)^{2}\right)-x f(t) .
\end{aligned}
$$

Here $m$ is the mass of the quantum particle while $m_{j}$ and $\omega_{j}$ refer to the mass and frequency of heat-bath oscillator $j$. In addition, $x$ and $p$ are the coordinate and momentum operators for the quantum particle and $q_{j}$ and $p_{j}$ are the corresponding quantities for the heat-bath oscillators. Also $f(t)$ is a c-number external force. The infinity of choices for the $m_{j}$ and $\omega_{j}$ give this model its great generality.

Use of the Heisenberg equations of motion leads to the QLE $[9,11]$

$$
m \ddot{x}+\int_{-\infty}^{t} d t^{\prime} \mu\left(t-t^{\prime}\right) \dot{x}\left(t^{\prime}\right)+V^{\prime}(x)=F(t)+f(t),
$$

where $V^{\prime}(x)=d V(x) / d x$ is the negative of the time-independent external force and $\mu(t)$ is the so-called memory function. $F(t)$ is the random (fluctuation or noise) operator force with mean $\langle F(t)\rangle=0$.

Thus, the coupling with the heat bath is described by two terms: an operator-valued random force $F(t)$ with mean zero, and a mean force characterized by a memory function $\mu(t)$. Explicitly,

$\mu(t)=\sum_{j} m_{j} \omega_{j}^{2} \cos \left(\omega_{j} t\right) \theta(t)$

with $\theta(t)$ the Heaviside step function. Also

$F(t)=\sum_{j} m_{j} \omega_{j}^{2} q_{j}^{h}(t)$

where $q^{h}(t)$ denotes the general solution of the homogeneous equation for the heat-bath oscillators (corresponding to no interaction). An exact solution can be obtained in the case of an oscillator potential $V(x)=\frac{1}{2} K x^{2}=\frac{1}{2} m \omega_{0}^{2} x^{2}$, which is best displayed as

$\tilde{x}(\omega)=\alpha(\omega)\{\tilde{F}(\omega)+\tilde{f}(\omega)\}$.

Here, the superposed tilde is used to denote the Fourier transform and $\alpha(z)$ is the generalized susceptibility (response function), which is given by

$\alpha(z)=\frac{1}{-m z^{2}-i z \tilde{\mu}(z)+K}$.

As already remarked, the BBR Hamiltonian is a special case of the IO model, for which [11]

$\operatorname{Re}\left[\tilde{\mu}\left(\omega+i 0^{+}\right)\right]=\frac{2 e^{2} \omega^{2}}{3 c^{3}} f_{k}^{2}$,

where the quantity $f_{k}$ is the electron form factor (Fourier transform of the electron charge distribution). In other words, we have allowed the electron to have structure.

The physically significant results for this model should not depend upon details of the electron form factor, subject, of course, to the condition that is be unity up to some large frequency $\Omega$ and falls to zero thereafter. A convenient form which satisfies this condition is 
$f_{k}^{2}=\frac{\Omega^{2}}{\omega^{2}+\Omega^{2}}$

Using this in (10), the Stieltjes inversion formula gives

$\tilde{\mu}(z)=\frac{2 s^{2} \Omega^{2}}{3 c^{3}} \frac{z}{z+i \Omega}$.

In addition, we found that the fluctuation force is $e \vec{E}$, where $\vec{E}$ is the electric field operator for the free BBR field. The above derivation started with the IO model and derived results for BBR as a special case. Actually, in our first paper on this subject [11], we dealt directly with the BBR Hamiltonian.

We emphasize again that our QLE given in (5) can be applied to many different heat baths of interest (the Ohmic, the single relaxation time model, the BBR and so on) but here we concentrate on the BBR. It turns out that the BBR model is unique in the sense that, as is well known, an essential aspect of QED theory is the necessity for mass renormalization. Thus the $m$ occurring in the QLE is actually the bare mass and the renormalized (observed) mass $M$ is given in terms of the bare mass $m$ by the relation [11]

$M=\frac{m+2 e^{2} \Omega}{3 c^{3}}=m+\tau_{e} \Omega M$,

where

$\tau_{e}=\frac{2 e^{2}}{3 M c^{3}} \simeq 6 \times 10^{-24} s$

In the next section, we will return to these results in order to express the QLE in terms of $M$ and hence obtain the equation of motion of a radiating electron. For now, we point out the importance of $\alpha(\omega)$ in that it leads us to a simple formula for $F_{0}(T)$, the free energy of the oscillator coupled to the radiation field, in the form (11)

$F_{0}(T)=\frac{1}{\pi} \int_{0}^{\infty} d \omega f(\omega, T) \operatorname{Im}\left\{\frac{d \log \alpha\left(\omega+i 0^{+}\right.}{d \omega}\right\}$,

where $f(\omega, T)$ is the free energy of a single oscillator of frequency $\omega$, given by

$f(\omega, T)=k T \log [1-\exp (-\hbar \omega / k T)]$.

This then led us to the conclusion [11] that the corresponding free energy level shift is given by

$\Delta F_{0}=\frac{\pi \alpha}{9 M c^{2}}(k T)^{2}$,

which in 3 dimensions is to be multiplied by 3 . It follows from thermodynamics [11] that the corresponding energy level shift is
$\Delta U_{0}=-\Delta F_{0}$

which is the negative of the result of what we regard as the flawed calculation given in (3) above. We conclude that our result given in (16) agrees with the results of the HollbergHall experiment and that the experiment actually measures free energy level shifts.

\section{Equation of motion of a radiating electron}

In a certain sense, the QLE given in (5) [in conjunction with (10) and the knowledge that $\vec{F}(t)=e \vec{E}$, where $\vec{E}$ is the elective field operator for the free BBR field] is the required equation of motion. However, as already noted, for BBR, the $m$ appearing in (5) is the rest mass and thus we must use (13) to get the corresponding result in terms of the observed mass $M$. This leads to the result [18]

$(m / \Omega) \dddot{x}(t)+M \ddot{x}(t)+V_{\mathrm{eff}}^{\prime}(x)=F_{\mathrm{eff}}(t)+f_{\mathrm{eff}}(t)$,

where

$f_{\mathrm{eff}}(t) \equiv f(t)+\Omega^{-1} f(t)$,

and similarly for the other "effective" quantities. We note that (19) is an exact quantum mechanical result. In the classical limit and with $V(x)=0$, we obtain

$M\left(\Omega^{-1}-\tau_{e}\right) \dddot{x}(t)+M \ddot{x}(t)=f(t)+\Omega^{-1} f(t)$.

We note the generality of this result in that we have not yet specified the cutoff frequency $\Omega$, which, of course, determines the form-factor. Also, the principle of causality (response due to an external force cannot precede the force) implies that the poles of the response function $\alpha(\omega)$ must lie in the lower half of the complex plane (noting that $\operatorname{Im} \omega>0[9,11]$ ) which, in turn, implies $m>0$. This leads to the conclusion [18] that

$\Omega<\tau_{e}^{-1}=1.60 \times 10^{23} s^{-1}$,

which rules out the possibility of a point electron [18] and explains why the Abraham-Lorentz equation is not an acceptable equation for the radiating electron [19]. In essence, we are now left with a family of solutions depending on the choice of $\Omega$ or, concomitantly, the choice of electron structure. The simplest solution emerges if we choose $m=0$ which to equivalent to choosing for $\Omega$ its largest permissible value of $\tau_{e}^{-1}$, corresponding to choosing the closest approach to a point electron consistent with causality. In that case, we obtain

$M \ddot{x}(t)=f(t)+\tau_{e} \dot{f}(t)$.

This is rather striking result in that it is only a second-order equation, it is correct to first order in $\tau_{e}$ and it is independent of the cutoff frequency $\Omega$. We note that the right-side of (23) 
depends only on the specified external force and thus it is a simple equation to solve. Finally, we note that other physically reasons choices for the form factor, as distinct from the choice given in (12), simply lead to additional higher-order terms in (23), such as $\tau_{e}^{2} \ddot{f}(t)$, as is shown explicitly in [18] and [19]. Also, we remark that the relativistic generalization of (23) has been obtained [20].

\section{Other miscellaneous applications}

The problem of Brownian motion is a special case of our general QLE given in (5) and corresponds to taking $f(t)=0$, $V(x)=0$, and $\mu\left(t-t^{\prime}\right)=2 m \gamma \delta\left(t-t^{\prime}\right)$, so that $\tilde{\mu}(\omega)=m \gamma$ where $\gamma$ is a constant. In addition, the classical high temperature limit is also assumed. The end result is that one obtains so-called normal (Einstein) diffusion with a diffusion constant $(k T / m \gamma)$, which is necessarily a classical result. However, for other choices of $\tilde{\mu}(\omega)$, one obtains anomalous diffusion, of interest in a variety of applications [21]. In the QED case, for which the choice for $\tilde{\mu}(\omega)$ given in (12) is relevant, interesting quantum effects are manifest. In particular, we find that, at $T=0$, the result for the diffusion constant contains not only quantum effects but also the bare mass appears in the result. In addition, it was possible to calculate the spreading of a wave packet in a BBR environment [21], a result not amenable to calculation by conventional QED methods.

Next, we point out that our result for the free energy, $F_{0}$, given in (15), provides the basis for calculating quantum effects on the laws of thermodynamics. In that context, we pointed out the flaws in a variety of papers which claimed that quantum effects could lead to violations of the second and third law of thermodynamics [22, 23, 24]. Moreover, we calculated explicitly quantum corrections for various thermodynamic quantities (free energy, energy, entropy, and specific heat) for a variety of heat bath models [25].

\section{Conclusions}

Treating the BBR field as a heat bath enabled us to treat it in the general context of stochastic physics, with its attendant powerful results (such as the fluctuation-dissipation theorem). As a result, we were able to calculate interesting physical phenomena not amenable to solution by the conventional techniques of atomic physics and QED.

\section{Acknowledgments}

It gives me great pleasure to dedicate this paper to Professor Walter R. Johnson, on the occasion of his retirement after 50 years at the University of Notre Dame. I entered the physics department at Notre Dame as a graduate student in 1958, the same year that Walter joined the faculty, and was honored to be the first student who started working with him. Not only did he patiently guide my Ph.D. studies but I also learned from him a basic knowledge in many areas (not the least being atomic physics and QED). I would also like to thank Professor G. W. Ford with whom I collaborated on all the work described here.

\section{References}

1. R. Brown, Philos. Mag. 4, 161 (1828); Ibid. 6, 161 (1829).

2. A. Einstein, Investigations on the Theory of the Brownian Movement (Dover, New York, 1956).

3. M. P. Langevin, "Sur la théorie du mouvement brownien," C. R. Acad, Sci., Paris 146, 530-533 (1908) (a translation of this article appears in D.S. Lemons, A. Gythiel, Am. J. Phys. 65 1079-1081 (1997)).

4. R. Nyquist, "Thermal Agitation of Electric Charge in Conductors," Phys. Rev. 32, 110-113 (1928).

5. J. B. Johnson, "Thermal Agitation of Electric Charge in Conductors," Phys. Rev. 32, 97-109 (1928).

6. H. B. Callen and T. A. Welton, "Irreversiblilty and Generalized Noise," Phys. Rev. 83, 34-40 (1951); G. W. Ford, J. T. Lewis, and R. F. O'Connell, 'Quantum Oscillator in a Blackbody Radiation Field II. Direct Calculation of the Energy using the FluctuationDissipation Theorem," Ann. Phys. (N.Y.) 185, 270-283 (1988).

7. G. W. Ford, M. Kac, and P. Mazur, "Statistical Mechanics of Assemblies of Coupled Oscillators," J. Math. Phys. 6, 504-515 (1965).

8. G. W. Ford and M. Kac, "On the Quantum Langevin Equation," J. Stat. Phys. 46, 803-810 (1987).

9. G. W. Ford, J. T. Lewis, and R. F. O'Connell, "The Quantum Langevin Equation," Phys. Rev. A 37, 4419-4428 (1988).

10. L. Hollberg and J. L. Hall, "Measurement of the Shift of Rydberg Energy Levels by Blackbody Radiation," Phys. Rev. Lett. 53, 230-233 (1984).

11. G. W. Ford, J. T. Lewis, and R. F. O'Connell, "Quantum Oscillator in a Blackbody Radiation Field," Phys. Rev. Lett. 55, 22732276 (1985).

12. G. W. Ford and R. F. O'Connell, ”Stark Shifts Due to Blackbody Radiation," J. Phys. B 19, L41-L46 (1986).

13. G. W. Ford, J. T. Lewis, and R. F. O'Connell, "On the Thermodynamics of Quantum - Electrodynamic Frequency Shifts," J. Phys. B 20, 899-906 (1987).

14. T. F. Gallagher and W. E. Cooke, "Interactions of Blackbody Radiation with Atoms," Phys. Rev. Lett. 42, 835 (1979).

15. J. W. Farley and W. H. Wing, "Accurate calculation of dynamic Stark shifts and depopulation rates of Rydberg energy levels induced by blackbody radiation. Hydrogen, helium, and alkalimetal atoms," Phys. Rev. A 23, 2397 (1981).

16. T. A. Welton, "Some Observable Effects of the QuantumMechanical Fluctuations of the Electromagnetic Field," Phys. Rev. 74, 1157 (1948).

17. X. L. Li, G. W. Ford, and R. F. O'Connell, "Energy Balance for a Dissipative System," Phys. Rev. E 48, 1547 (1993).

18. G. W. Ford and R. F. O'Connell, "Radiation Reaction in Electrodynamics and the Elimination of Runaway Solutions," Phys. Lett. A 157, 217 (1991).

19. R. F. O'Connell, 'The Equation of Motion of an Electron," Phys. Lett. A 313, 491 (2003).

20. G. W. Ford and R. F. O'Connell, "Relativistic Form of Radiation Reaction," Phys. Lett. A 174, 182(1993).

21. G. W. Ford and R. F. O'Connell, Anomalous diffusion in quantum Brownian motion with colored noise," Phys. Rev. A 73, 032103 (2006).

22. G. W. Ford and R. F. O'Connell, Entropy of a Quantum Oscillator coupled to a Heat Bath and implications for Quantum Thermodynamics," Physica E 29, 82 (2005). 
23. G. W. Ford and R. F. O'Connell, A Quantum Violation of the Second Law," Phys. Rev. Lett. 96, 020402 (2006).

24. R. F. O'Connell, Does the Third Law of Thermodynamics hold in the Quantum Regime?," J. Stat. Phys. 124, 15 (2006).

25. G. W. Ford and R. F. O'Connell, Quantum thermodynamic functions for an oscillator coupled to a heat bath, Phys. Rev. B, 75, 134301 (2007) 